\title{
Sistema fiscal y redistribución: la transición fiscal española $(1960-1990)^{1}$
}

\author{
Sara Torregrosa Hetland* \\ Universidad de Lund
}

\section{RESUMEN}

La tesis analiza el sistema fiscal español entre los años 1960 y 1990, con especial atención a sus efectos en la distribución de la renta. Se abordan las reformas que tuvieron lugar durante la transición a la democracia, aportando una evaluación cuantitativa consistente de los cambios.

A causa de la larga dictadura sufrida por el país entre 1936/39 y 1976, España introdujo las ideas fiscales del siglo XX con considerable retraso respecto de sus vecinos, manteniendo largamente un sistema de impuestos bajos, regresivos e ineficientes. Con el retorno de la democracia, diversas leyes de reforma fiscal pretendieron hacer el sistema más progresivo y capaz de generar mayores ingresos públicos, para la financiación de un incipiente Estado del Bienestar.

El principio de progresividad, sin embargo, tuvo una aplicación práctica limitada. Los cálculos de la tesis lo muestran así, estimando la distribución resultante de la carga fiscal, la persistencia en las desigualdades de renta, y el fuerte impacto del fraude en la imposición personal.

La interpretación ofrecida se sitúa entre el legado de la dictadura en la configuración del régimen parlamentario y el nuevo contexto económico, que combinaba la ralentización del crecimiento con una mayor apertura y movilidad internacional del capital.

Palabras clave: desigualdad, reforma fiscal, redistribución, progresividad, democratización. Códigos JEL: H20, D31, N34

\section{Abstract}

This thesis analyses the Spanish tax system between 1960 and 1990, with special attention to its effects on the income distribution. It provides with a quantitative consistent evaluation of the reforms that took place during transition to democracy.

Because of the long dictatorship suffered by the country between 1936/39 and 1976, Spain introduced the 20th Century tax ideas with a considerable lag with respect to its neighbours. Taxes were low, regressive and inefficient. With the return of democracy, several tax reform laws intended to make the system more progressive and able to generate increased public revenue, in order to fund an emerging Welfare State.

*sara.torregrosa-hetland@ekh.lu.se 
The principle of progressivity, however, had limited practical application. The calculations of the thesis show this by estimating the resulting distribution of the tax burden, the persistent levels of income inequality, and the strong impact of tax evasion in personal income taxation.

The offered interpretation looks at the legacies of the dictatorship on the institutional design of the parliamentary regime, and the new economic context, combining lower growth and increased openness and international capital mobility.

Keywords: inequality, tax reform, redistribution, progressivity, democratization.

JEL Codes: H20, D31, N34

\section{INTRODUCCIÓN}

a investigación sobre el Estado del Bienestar es un área fructífera de las ciencias sociales. Se han debatido las evoluciones de la legislación protectora, los sistemas de seguros sociales, los programas de pobreza, y la desigualdad. Diversas teorías han intentado explicar los determinantes de dichos desarrollos, desde los cambios sociales producidos por la industrialización (Wilensky, 2002) hasta el corporativismo, la democratización, o la movilización de la clase trabajadora (Esping-Andersen, 1990; Lindert, 1994; Korpi y Palme, 1998).

A los sistemas fiscales, no obstante, se les ha prestado relativamente menor atención, a pesar de su papel esencial en la recaudación de los fondos necesarios para las funciones públicas, y de tener también en sí mismos, claramente, consecuencias distributivas. El crecimiento de la presión fiscal se ha relacionado con los efectos de la guerra (Peacock y Wiseman, 1961; Scheve y Stasavage, 2012), así como con el uso de técnicas impositivas más eficientes (los impuestos personales sobre la renta, después los del valor ańadido; véase p. ej. Lindert, 2004). Estas innovaciones fiscales estarían estrechamente conectadas a desarrollos sociales y económicos que permitieron reducir los costes de recaudación, como la alfabetización (Aidt y Jensen, 2009a), las tecnologías de la información, o el tamaño empresarial (Kleven, Kreiner, y Saez, 2015).

Los impuestos también han sido vinculados a la extensión de derechos políticos: pueden ser entendidos como un reflejo de los equilibrios de poder, donde diferentes grupos sociales intentan desplazar la carga hacia otros (Mares y Queralt, 2015), o como parte de un 
contrato por el cual los contribuyentes adquieren cierta influencia sobre el estado al que financian (Timmons, 2005). La reciente "New Fiscal Sociology" intenta tender puentes entre la investigación en impuestos y otros campos de la historia social, económica y política (Martin, Mehrotra, y Prasad, 2009).

Dentro de este marco general, la tesis de la autora explora los cambios en el sistema impositivo que acompańaron a la transición a la democracia en Espańa., ofreciendo un análisis cuantitativo de los efectos de la reforma sobre la distribución de la renta y la financiación del Estado del Bienestar. Subyace la idea de leer en el funcionamiento de la fiscalidad algunos rasgos de la reforma política. ¿Cómo afectó la democracia a los impuestos que pagaban los españoles? ¿Llevó el incremento de la igualdad política a un incremento similar en la igualdad económica? ¿Qué nivel de cumplimiento tuvieron los ciudadanos con el nuevo sistema? Y, dados los limitados resultados obtenidos, ¿cuáles fueron los obstáculos a la progresividad y la redistribución de la renta en los inicios del periodo democrático?

Nuestro caso se enmarca dentro de la literatura sobre los efectos distributivos de las transiciones, que normalmente ha señalado teóricamente hacia un descenso de la desigualdad, debido a un incremento del papel redistributivo del estado. Así lo implica el modelo clásico de Meltzer y Richard (1981), seguido en lo esencial por aportaciones más recientes como Acemoglu y Robinson (2001) y Boix (2003), si bien reconociendo la existencia de posibles limitaciones en el propio proceso de transición. Los trabajos empíricos no alcanzan conclusiones unánimes (Gradstein y Milanovic, 2004; Albertus y Menaldo, 2014; Aidt y Jensen, 2009b). Un enriquecimiento de la literatura pasaría por considerar limitaciones en la igualdad de la representación política de los ciudadanos (Acemoglu y Robinson, 2008; Ardanaz y Scartascini, 2013), o la consideración de las actitudes sobre fiscalidad, que se suelen dar por sentadas respecto a la búsqueda del interés propio inmediato, cuando la realidad parece ser bastante más compleja (Harms y Zink, 2003; Grüner y Corneo, 2000; Giuliano y Spilimbergo, 2009). Las preferencias aparecen limitadas por el nivel de conocimiento sobre la 
realidad social (Cruces, Perez-Truglia, y Tetaz, 2013; Bartels, 2005) o la confianza en el gobierno (Kuziemko, Norton, Saez, y Stantcheva, 2013; Svallfors, 2013).

El resto del texto se organiza de la forma siguiente: la sección 2 describe los procesos asociados de democratización y reforma fiscal ocurridos en Espańa entre 1977 y 1986. Posteriormente se presentan los resultados empíricos principales de la tesis: la evolución de la desigualdad en el apartado 3, los efectos redistributivos del presupuesto público en el 4, y el impacto del fraude en el 5. La sección 6 cierra, con una referencia a la investigación sobre los determinantes del resultado alcanzado.

LA TRANSICIÓN ESPAÑOLA A LA DEMOCRACIA Y LA REFORMA FISCAL DE I977-I986

El retorno de España a la democracia en los años 1970 ha sido abordado en una abundante literatura, haciendo referencia a las teorías internacionales y a la "tercera ola" de democratización. Se ha discutido el papel del desarrollo económico, las élites y la oposición de base en el proceso (Molinero y Ysàs, 2008; Saz, 2010). La interpretación más extendida es que la transición a la democracia fue el resultado de la incapacidad de ambos bandos (continuistas, rupturistas) para tomar un control completo de la situación, dando lugar a una "reforma pactada" donde el nuevo diseño institucional se desarrolló sin ruptura revolucionaria para con el marco legal de la dictadura (Colomer, 1998). Como es bien sabido, el proceso fue orquestado por Adolfo Suárez y sus principales hitos fueron la Ley de Reforma Politica de 1976, las elecciones democráticas de junio de 1977, y la aprobación de una nueva constitución en diciembre de 1978. Los partidos de la oposición lograron el proceso constituyente y la legalización plena (aunque tardía) de los grupos de la izquierda, mientras que los viejos poderes de la oligarquía mantuvieron una cámara alta (el Senado) y lograron un sistema electoral sesgado hacia las opciones conservadoras (Lago y Montero, 2005).

El sistema fiscal español del periodo ha sido estudiado por trabajos anteriores, con análisis de los cambios en los ingresos (Comín, 
2007; Pan-Montojo, 1996) y en los gastos (Espuelas, 2013), pero sin una estimación cuantitativa de los impactos sobre la desigualdad. Una profunda reforma se inició en 1977, con los objetivos de aumentar los ingresos directos y modernizar el sistema, para financiar un incipiente Estado del Bienestar. La impulsó el primer ejecutivo de la nueva democracia, presidido por Suárez y formado por miembros de la nueva coalición, y después partido, Unión de Centro Democrático. Este era un grupo heterogéneo en el que coexistían liberales, demócrata-cristianos y socialdemócratas. La reforma fiscal fue el proyecto del ala izquierda del partido, donde era una prioridad - no compartida en sus desarrollos por otros miembros prominentes del grupo.

Las líneas maestras de la reforma habían sido sugeridas en las décadas anteriores por destacados economistas del país, liderados por Enrique Fuentes Quintana. Varias propuestas del Instituto de Estudios Fiscales $(1973 ; 1976)$ pretendieron impulsar la adopción en España de un modelo fiscal europeo, que permitiera una distribución de la carga más progresiva y elevara los ingresos, homologando al país con el núcleo de la Comunidad Económica Europea. Quedó patente, no obstante, que dicha reforma sólo podría tener lugar bajo un sistema democrático: al igual que en etapas anteriores de la historia nacional, los cambios fiscales fueron asociados a profundos cambios políticos (Fuentes Quintana, 1990). En el gobierno de Suárez de 1977, Fuentes Quintana fue nombrado Vicepresidente y Ministro de Economía, mientras que su colaborador Francisco Fernández Ordóńez, un destacado socialdemócrata, ocupó la cartera de Hacienda.

Los Pactos de la Moncloa de noviembre de 1977 incluyeron algunos puntos de reforma fiscal progresiva, como contrapeso a los efectos distributivos negativos de la estabilización económica que emprendió el gobierno (Fuentes Quintana, 2004; Comín, 2007). El mismo mes, la primera ley del nuevo parlamento introdujo el impuesto sobre el patrimonio, incluyendo también una amnistía fiscal y diversas medidas anti-fraude (destacando la abolición del secreto bancario y la introducción de la figura del delito fiscal). La piedra angular de la reforma apareció en septiembre de 1978 con el Im- 
puesto sobre la Renta de las Personas Físicas (reemplazando a todo un abanico de impuestos de producto), que debía convertirse en el pilar fundamental de la recaudación, acorde con las ideas sobre equidad fiscal del siglo XX. Con estos cambios, la imposición directa se puso al nivel de la indirecta en los presupuestos públicos (véase la figura 1): un objetivo explícito de la reforma, pero insuficiente para llevar a conclusiones sobre la progresividad global del sistema.

Figura 1: Principales categorías en los ingresos fiscales españoles y de la UE-14, como porcentaje del PIB

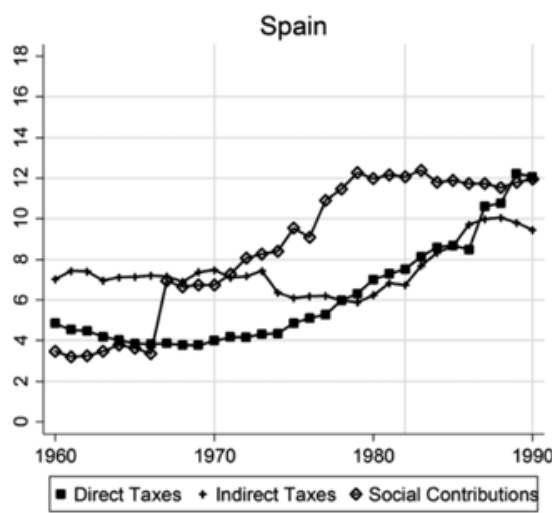

EU-14

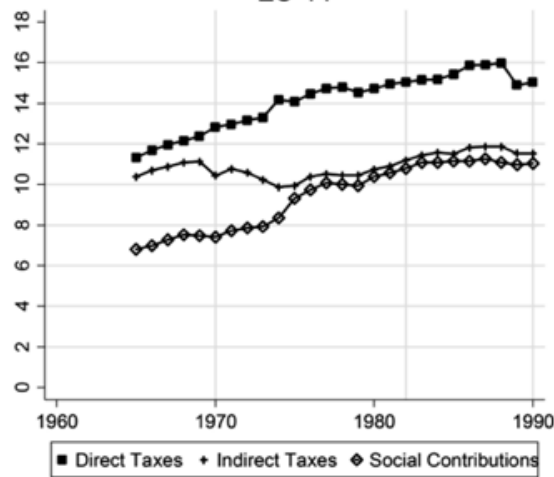

Fuente: España, Cuentas de las Administraciones Públicas, Ministerio de Hacienda. UE14, OECD Statistics (media no ponderada de los países de la UE-15 salvo Espańa). Hay una ruptura en la serie española en 1967, debido a la entrada en vigor de la ley de la Seguridad Social de 1963, que implicó también cambios en la contabilidad. 
El historiador Pan-Montojo (1996) identificó un periodo de "contra-reforma fiscal" tras estos primeros desarrollos. La reforma estuvo relacionada con la crisis del partido gobernante UCD, llevando a su división y pérdida de apoyo electoral ${ }^{2}$. La primera etapa de consenso político dio paso a una oposición más enconada tras la aprobación de la Constitución y de cara a las nuevas elecciones de 1979. De este modo, los siguientes proyectos se vieron retrasados y no llegaron a aprobarse en el parlamento (como relata también Comín, 2007). La falta de capacidad administrativa y el obstruccionismo de las instituciones financieras dificultaron la aplicación plena de la imposición personal, y la reforma prevista en la indirecta se retrasó. Las elecciones de 1982 llevaron al poder al partido socialista, que se mantendría hasta 1996, culminando algunos aspectos del plan - si bien ya en un contexto ideológico diferente (en el cual el apoyo a la idea de la progresividad había disminuido notablemente), y con un marco internacional también distinto. El IVA se introdujo en 1986, en el momento de entrada en la CEE.

La Seguridad Social es parte de la misma historia. Bajo el Franquismo, se había establecido un sistema Bismarckiano en 1963-67 (Comín, 2010), que incrementó fuertemente la presión sobre las rentas del trabajo (convirtiéndose las cotizaciones sociales en la mayor fuente de ingresos públicos a principios de los setenta). El sistema era enormemente complejo y regresivo, y su reforma e integración en el sistema general de ingresos se planteaba también durante la transición - pero no recaía sobre el mismo ministerio, y se vio dificultado por las resistencias a incrementar los costes laborales de las empresas. El modelo contributivo sólo vio cambios menores hasta finales de los ochenta.

Tras 1978, los presupuestos públicos experimentaron una considerable expansión, que permitió desarrollos en el Estado del Bienestar (si bien, coexistiendo con la generación de déficits e ingresos por seńoreaje). El proceso de convergencia con el "modelo europeo" no obstante quedó incompleto, como muestra el diferencial persistente en la figura 1, especialmente en cuanto a los impuestos directos. Este diferencial se amplió de nuevo con las crisis económicas de los noventa y de 2008, apuntando a la vulnerabilidad del proceso. 
Tras 1990, el año final de nuestro estudio, no ha habido un nuevo rediseño global del sistema impositivo, aunque sí numerosas modificaciones parciales, y un destacado proceso de descentralización. Nuestra atención, no obstante, se centra en los primeros desarrollos de la democracia. ¿Hubo una transición hacia la progresividad como resultado de los cambios mencionados? A pesar de los objetivos explicitados en la reforma, y de los modelos clásicos de la economía política, el elevado peso de la tributación sobre el trabajo en el sistema resultante sostiene nuestra hipótesis de una incidencia todavía regresiva o como mucho proporcional a la altura de 1990 (como en Manresa y Calonge, 2001).

\section{LA EVOLUCIÓN DE LA DESIGUALDAD}

El consenso general en la literatura económica española ha sido largamente que la desigualdad de la renta se redujo de manera notable durante los años de la transición a la democracia (Alcaide, 2000; Ayala, Jurado, y Pedraja, 2006). Ello tendría que ver tanto con la evolución de la estructura económica y la distribución de las rentas del mercado, como con el desarrollo del estado del bienestar y la redistribución pública. Dicho relato cuadra bien con las expectativas de reducción de las disparidades sociales como consecuencia de la democratización.

La fuente histórica fundamental para estudiar la distribución de la renta en este periodo son las Encuestas de Presupuestos Familiares de 1973-74, 1980-81 y 1990-91 (en adelante, EPFs). Pese a su innegable utilidad y riqueza, es necesario reconocer que adolecen de graves sesgos, que llevan a infravalorar la desigualdad. El problema fue reconocido por el propio Instituto Nacional de Estadística y algunos de los estudios anteriores, como Alcaide y Alcaide (1974); Pena y Callealta (1996) u Oliver (1997). El sesgo tendrá, además, intensidades diferentes según la participación en la renta total de las procedentes de cada fuente (trabajo, capital, mixtas).

En la tesis llevo a cabo una corrección de los datos individuales, ajustando a la postre al marco dado por la Contabilidad Nacional. El ejercicio se basa en planteamientos frecuentes en América Latina 
(CEPAL, 1991; Engel, Galetovic, y Raddatz, 1999; Barreix, Bès, y Roca, 2009), y que recientemente se han reintroducido en los estudios sobre la desigualdad en los países desarrollados (Neri y Zizza, 2010; Fixler y Johnson, 2012).

El resultado final del ajuste de los microdatos son unos niveles de desigualdad más elevados y considerablemente persistentes en el periodo. Así, los elementos distributivos positivos de la transición (desarrollo de las transferencias sociales y reducción de la regresividad fiscal) habrían sido "compensados" por evoluciones negativas en otros componentes de la renta (vinculados a la reestructuración económica, la apertura internacional y el incremento del desempleo).

Crítica y ajuste de las fuentes: las Encuestas de Presupuestos Familiares Las EPFs aportan información valiosa sobre características socioprofesionales de las familias, sus gastos en distintos bienes y servicios, y su renta disponible ${ }^{3}$. La limitada fiabilidad de los datos de renta ha sido reconocida (al igual que en otros países), por diversos motivos; tendiendo los sesgos identificados a una infra-valoración de la desigualdad (Garde, Martínez, y Ruiz-Huerta, 1996). En la tesis, se ha realizado una corrección de uno de los sesgos: el derivado de la infra-declaración de rentas por parte de los hogares (debida a deficiencias en el control contable, reticencias a compartir esta información, etc).

Como muestra de la extensión del problema, una simple comparación de los datos de ingresos y gastos familiares nos muestra que sólo un 30-40\% de los hogares declararon gastar cantidades iguales o inferiores a su renta anual, mientras en torno a un $10 \%$ consumían más del doble de dicho nivel. Esto es altamente improbable, máxime cuando la Contabilidad Nacional (CNE) proporciona valores positivos al ahorro de los hogares en dichos años. El marco de la CNE se suele considerar más fiable para el agregado, y las rentas disponibles totales declaradas en las EPFs estaban sólo en torno al 70\% de las de la CNE. Dicha discrepancia es más elevada para las rentas no salariales, si bien decreciente con el paso de las décadas. 
El trabajo empírico sigue las ideas aportadas por Alcaide y Alcaide (1974) y Pena y Callealta (1996) en cuanto a la explotación de las diferencias entre gasto e ingreso y el ajuste a la CNE, pero realiza cálculos específicos diferentes. En primer lugar, me baso en la propuesta de Pissarides y Weber (1989), adaptada en su interpretación por Martí nez-López (2013), para estimar un coeficiente de infradeclaración de los autónomos relativo al de los trabajadores por cuenta ajena. En segundo lugar, llevo a cabo un ajuste a las rentas de la Contabilidad Nacional, de modo diferenciado para cada tipo de renta (como fue sugerido ya por Oliver, 1997).

El primer cálculo se basa en la estimación de una curva de Engel, en la cual se utiliza el gasto en alimentación de los hogares como variable dependiente, y entre las explicativas se introduce una variable dummy para detectar un aparente mayor gasto de las familias de trabajadores autónomos. Siendo positivo y significativo el coeficiente de esta variable, se puede interpretar que nos está indicando la existencia de infra-declaración de rentas en estos hogares (cuya renta real sería la misma que la de un hogar de trabajadores por cuenta ajena con el mismo gasto en alimentación y otras características similares).

Los detalles de la estimación se pueden consultar en Torregrosa Hetland (2016). Para este resumen, nos quedamos con el resultado básico: los autoempleados estarían infra-declarando sus rentas en un $14-20 \%$ más que los asalariados. Retenemos este coeficiente para integrarlo en el siguiente ejercicio.

Seguidamente, comparamos los totales de cada tipo de renta en las EPFs con los presentes en la Contabilidad Nacional (sector hogares). Se ha trabajado con cuatro tipos de renta diferentes, procedentes del trabajo asalariado, el auto-empleo, el capital, y las transferencias recibidas por los hogares. El cálculo general es una ratio renta $C$ $N E^{\text {renta }} E P F$ para cada tipo, deduciendo previamente de la primera magnitud los impuestos correspondientes (ya que las cantidades en las encuestas son en términos netos). Para los detalles del cálculo, nuevamente nos remitimos a la versión publicada del estudio.

La tabla 1 muestra los factores de ajuste obtenidos con la combinación de ambos métodos ${ }^{4}$. Como se puede observar, tienden a 
decrecer con el tiempo, apuntando aparentemente a una creciente fiabilidad de las encuestas; la excepción a este patrón general son las rentas del capital mobiliario. Estos factores se aplican a continuación a los datos individuales. Por efecto de la composición cambiante de los ingresos de los hogares, el ajuste al alza resulta tener forma de J, siendo menor en la parte central de la distribución y alcanzando sus niveles más altos en las familias más acomodadas.

Tabla 1: Factores de corrección por tipo de renta

\begin{tabular}{cccc}
\hline & 1973 & 1980 & 1990 \\
\hline Salarios & 1,35 & 1,19 & 1,12 \\
\hline Auto-empleo \& Alquileres & 1,63 & 1,51 & 1,31 \\
\hline Capital mobiliario & 4,35 & 3,40 & 6,85 \\
\hline Transferencias privadas & 2,51 & 2,05 & 4,02 \\
Prestaciones sociales & & & 1,43 \\
\hline \hline
\end{tabular}

Fuente: Estimación propia, Torregrosa Hetland (2016).

\section{RESULTADOS: LA PERSISTENCIA DE LA DESIGUALDAD}

Los niveles de desigualdad resultantes de la corrección se muestran en la tabla 2. El indicador utilizado es la renta disponible total de los hogares, debido a la comparabilidad con los cálculos de Alcaide, el primer investigador que abordó esta problemática ya contemporáneamente a las encuestas. Como se puede observar, el ajuste de las rentas supone un aumento de la desigualdad (salvo en 1980, cuando no hay diferencia estadística), y un cambio en la tendencia observada. Los datos originales de las EPFs muestran una caída de tres puntos en el índice de Gini durante estos veinte años, mientras que Alcaide (2000) elevó esta diferencia a diez puntos. La metodología seguida por éste, no obstante, no es homogénea a lo largo de los años (y se basa en una encuesta de características diferentes para 1990). Con el procedimiento descrito en este trabajo, el descenso de la desigualdad evidenciado durante la primera mitad del periodo se detiene al llegar a 1980. 
Tabla 2: La desigualdad de la renta en distintos trabajos

\begin{tabular}{llll}
\hline & 1973 & 1980 & 1990 \\
\hline EPFs originales & 36,2 & 34,3 & 33,0 \\
Corrección de Alcaide (2000) & 44,6 & 36,3 & 34,7 \\
Torregrosa Hetland (2016) & 36,8 & 33,5 & 34,8 \\
\hline \hline
\end{tabular}

Fuente: Alcaide (2000) y cálculos de la autora.

Gini de la renta disponible total de los hogares. El dato de Alcaide para 1990 se basa en la Encuesta Continua de Presupuestos Familiares.

Cuando nos concentramos en la desigualdad entre individuos, empleando los Ginis de la renta equivalente, el nivel es ligeramente inferior ${ }^{5}$. Los valores que obtengo con los microdatos ajustados son, respectivamente, 34,6, 32,6 y 33,0 (mientras que los de las rentas sin ajustar eran 32,6, 32,0 y 30,0). Las tendencias son las mismas: descenso muy ligero de la desigualdad, que se detiene en los años finales.

Esta considerable persistencia en los niveles de desigualdad coexiste, no obstante, con interesantes cambios distributivos cuando miramos más allá, en otros indicadores. Durante este periodo operaron varias fuerzas en direcciones contrarias, que se acabaron compensando entre sí. Por una parte, el incremento de las transferencias públicas a los hogares y la introducción de la progresividad en el sistema fiscal (como veremos en el siguiente apartado); por otra, una mayor desigualdad en las rentas del trabajo (debido al aumento del desempleo) y la creciente participación de las rentas del capital ${ }^{6}$.

Asimismo, podríamos enriquecer la descripción con atención a los niveles de renta, cuya desigualdad en términos absolutos crecía al tiempo que se mantuvo constante en términos relativos, $o$ a los indicadores de extracción à la Milanovic, Lindert, y Williamson (2011). Ambos aspectos se exploran con mayor detalle en la tesis y en un trabajo actualmente en curso ${ }^{7}$.

En suma, tras el ejercicio de ajuste de las encuestas, la desigualdad aparece prácticamente constante en las décadas contempladas. Esto sugiere que, en España, la democratización no fue una fuerza suficientemente poderosa como para limitar las diferencias de renta existentes. También implica, en el aspecto 
metodológico, que la infra-declaración debe ser tenida en cuenta en los estudios sobre la distribución de la renta y sus cambios en el tiempo, especialmente cuando hay cambios significativos en la distribución factorial.

\section{EFECTOS EMPÍRICOS DEL SISTEMA FISCAL}

El ejercicio central de la tesis es una estimación de la distribución de la carga fiscal en el país, para analizar cómo evoluciona a través de las reformas. El trabajo se inserta en una larga tradición internacional, iniciada a mediados del siglo XX con los trabajos de Barna (1945) y Musgrave (1951), y continuada hasta la actualidad p.ej. por Piketty y Saez (2007) y Breceda, Rigolini, y Saavedra (2009). En España, existen estudios sobre impuestos específicos (sobre todo el IRPF; Onrubia, Rodado, Díaz, y Pérez, 2007), y algunos generales (destacando Perona, 1972 y Manresa y Calonge, 2001). Pero todavía no contábamos con una estimación de medio-largo plazo realizada con una aproximación metodológica consistente, que permita contrastar las evoluciones fiscales con las del régimen político.

Como veremos, el resultado principal es que la regresividad no fue erradicada del sistema, aunque sí atenuada, tras haberse incrementado probablemente durante los años sesenta. Así, la imposición efectuaba una redistribución inversa de la renta, contradiciendo el discurso político y la demanda al respecto de la ciudadanía. Los niveles de redistribución total alcanzados quedaron lejos de la convergencia con el modelo europeo de Estado del Bienestar.

\section{Metodología del ANÁlisis de DistribuCión DE LA CARGA}

El trabajo se realiza mediante la imputación de los ingresos fiscales del año a los grupos sociales que habrían soportado la correspondiente carga económica. Para ello, sigo los resultados de una amplia literatura previa, teórica y empírica, sobre incidencia impositiva. Algunas limitaciones de este tipo de estudios son la incertidumbre sobre la incidencia de algunos impuestos y la no consideración de efectos dinámicos. 
- Marco temporal: se analizan los ańos 1970, 1982 y 1990 como representativos de la evolución general. La elección se ha basado en la disponibilidad de los datos necesarios ${ }^{8}$. Se utiliza la perspectiva anual (la más habitual en la literatura), sin tomarse las opciones relacionadas con la renta permanente o la imposición total en el ciclo vital de los individuos.

- Se imputan los ingresos fiscales de las Administraciones Públicas, en torno a un $90 \%$ (la desagregación que permiten los datos), incluyendo cotizaciones sociales ${ }^{9}$.

- Hipótesis de incidencia: se utilizan las más comunes en la literatura internacional. Es decir, los impuestos sobre la renta recaerían en el contribuyente nominal, los impuestos indirectos se trasladarían al consumidor, y las cotizaciones sociales al trabajador. Para el impuesto de sociedades (dada la inexistencia de consenso) se ha aplicado una hipótesis mixta con participación al 33\% entre los capitalistas, los trabajadores y los consumidores. Se han realizado también cálculos alternativos para varios impuestos con dudas sobre su incidencia (véase en la tesis).

- Los hogares se ordenan según su renta antes de impuestos (remuneraciones de los factores de producción en el mercado $)^{10}$, utilizando siempre rentas equivalentes (escala OCDE) y ponderación individual (es decir, nos interesa obtener resultados referentes a desigualdad y redistribución entre personas). La fuente base para ello son las EPFs ajustadas a Contabilidad Nacional en el ejercicio anterior.

- La imputación se realiza utilizando la distribución de la base impositiva entre los hogares: se asume implícitamente, por tanto, que el fraude está homogéneamente distribuido. Esto introduce un sesgo esperado de infra-estimación de la regresividad.

- Índices utilizados en la literatura internacional: tipos medios efectivos, índice de Kakwani para la progresividad, índice de Reynolds-Smolensky para la redistribución ${ }^{11}$. El primer indicador mostrará progresividad si crece con la renta; los dos últimos si se obtienen signos positivos. Para todos los cálculos, la base es la renta bruta de los hogares. 
RESULTADOS: REGRESIVIDAD ATENUADA

El gráfico 2 muestra los tipos medios efectivos estimados para 1970, 1982 y 1990, para el conjunto de los impuestos. Como se puede observar, los tipos soportados por los hogares eran más altos para las familias más pobres: se estima un 28\%, 44\% y $70 \%$ de presión fiscal total para la decila segunda en cada ańo, frente a 20\%, 35\% y $46 \%$ en la superior. Si nos fijamos en el famoso top 1\%, esta conclusión se intensifica: los tipos efectivos pagados estaban en torno al $16 \%$ en $1970,32 \%$ en 1982 y $44 \%$ en 1990 .

Este resultado es producto de la composición de la presión impositiva total, donde tienen un peso fundamental las cotizaciones sociales y los impuestos indirectos. La regresividad de la imposición sobre el consumo es un rasgo bien conocido, apenas mitigado por aspectos como los tipos diferenciados existentes en el IVA, y que resulta más intensa cuanto mayor es la desigualdad de rentas existente en la base. Asimismo, pesan más sobre las clases mediasbajas las cotizaciones sociales, al estar distribuidas sobre las rentas del trabajo con exenciones para los salarios altos (por efecto del modo de cálculo, hasta 1972 utilizando bases homogéneas por categorías laborales y después limitando por unos topes máximos la base imponible).

Los impuestos directos, por otra parte, se comportaban de manera progresiva ya en 1970 , si bien con tipos muy reducidos (del 4 al 6\%). Para 1982 y 1990, los tipos directos en la decila superior ya habían pasado al $12 \%$ y $21 \%$ respectivamente. Esto muestra los efectos de la reforma aquí analizada, pero también su insuficiencia, una vez combinados todos los componentes del sistema resultante.

Los índices de progresividad y redistribución de la figura 3 así lo muestran: sus signos son siempre negativos, aunque la regresividad se redujo. El peso de los impuestos directos (dado por sus tipos medios) era insuficiente para compensar los demás componentes. Así, en su conjunto, el sistema impositivo efectuaba una redistribución inversa, no sólo en 1970 pero también en 1982 y 1990, tras la consolidación del régimen parlamentario. 
Figura 2: Tipos Medios Efectivos, 1970 a 1990

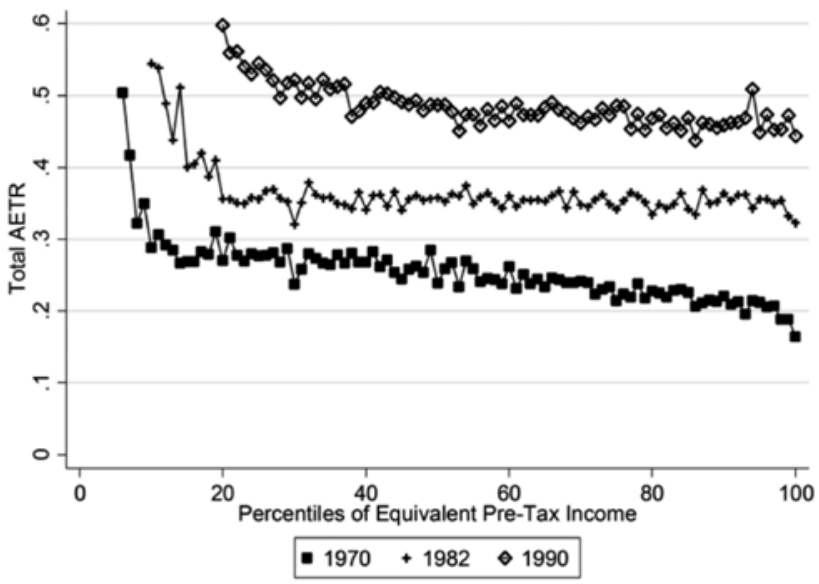

Fuente: Cálculos de la autora (Torregrosa Hetland, 2015b).

Percentiles de individuos ordenados por su renta bruta equivalente. Tipos medios efectivos de la suma de todos los impuestos.

Figura 3: Índices de progresividad y redistribución

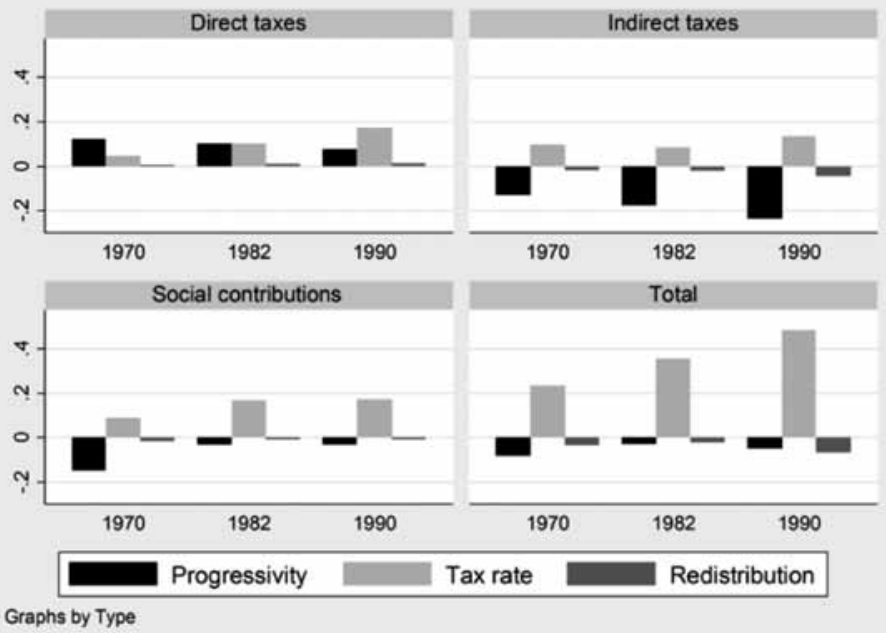

Fuente: Estimación propia, Torregrosa Hetland (2015b).

Progresividad: índice de Kakwani. Redistribución: índice de Reynolds-Smolensky (diferencia entre el Gini antes y después de aplicar el impuesto). Para la ordenación se ha utilizado la renta bruta equivalente, con ponderación por individuos. 
La tabla 3 muestra los índices de Gini de las distintas fases de la renta. La diferencia entre los correspondientes a las rentas brutas y netas iguala el índice de Reynolds-Smolensky mostrado arriba: impacto negativo del sistema impositivo sobre la desigualdad (pese a los efectos parciales positivos de los impuestos directos). Por supuesto, ello no implica que el efecto general del sector público sobre las clases populares fuera extractivo: el desarrollo del Estado del Bienestar fue la otra cara de la moneda de la creciente capacidad fiscal. Se incrementaron las prestaciones sociales en transferencias a los hogares, así como en los sistemas públicos de sanidad y educación. Así, el lado del gasto permitió mejoras en la distribución del bienestar económico y probablemente hacia una mayor igualdad de oportunidades. El impacto de las transferencias monetarias a las familias aparece aquí como la diferencia entre las columnas 2 y 3 (provocando una reducción en el índice de Gini de unos 4 puntos en 1970, y de cerca de 8 en 1982 y 1990). Por desgracia, no contamos con estimaciones consistentes del efecto de los servicios públicos en especie (sí las hay en otros trabajos, como p.ej. Bandrés, 1993; Calero, 2001).

Tabla 3: Desigualdad de la renta a través del sistema fiscal (índice de Gini)

\begin{tabular}{cccc}
\hline & $\begin{array}{c}\text { Rentas brutas del } \\
\text { mercado }\end{array}$ & $\begin{array}{c}\text { Rentas después de } \\
\text { impuestos }\end{array}$ & $\begin{array}{c}\text { Rentas después de } \\
\text { impuestos y } \\
\text { transferencias }\end{array}$ \\
\hline 1970 & 38,0 & 41,5 & 36,2 \\
1982 & 42,1 & 44,5 & 34,5 \\
1990 & 42,5 & 49,2 & 37,3
\end{tabular}

Fuente: Estimación propia, Torregrosa Hetland (2015b).

Rentas equivalentes en todos los casos, con ponderación por individuos y ordenación según las rentas brutas.

El efecto global del sistema de impuestos y transferencias se aproxima comparando las rentas brutas con las de la última columna, que representa la desigualdad finalmente existente en términos de capacidad de consumo neta (no confundir con la renta disponible). Globalmente, se producía un efecto positivo, reductor de la desigualdad, en 1,7, 7,5 y 4,5 puntos de Gini respectivamente en 
1970, 1982 y 1990. El sector público impidió que el incremento de la desigualdad en las rentas del mercado se trasladase por completo a las netas. Pero la redistribución no se hizo a través de los impuestos, no extrajo elevadas contribuciones de la élite, y no llevó a una reducción efectiva de las desigualdades.

\section{El FRAUDE EN EL IMPUESTO PERSONAL SOBRE LA RENTA}

El fraude ha sido largamente un problema destacado en el sistema fiscal español (Comí n, 1994). También fue considerado por los contemporáneos uno de los aspectos de menor éxito en la reforma de la transición, pese al énfasis realizado en este aspecto por el ministro Fernández Ordóńez. La persistencia del fraude impediría la culminación de las ideas fiscales del siglo XX, en cuanto a la base de la imposición en la capacidad contributiva de los individuos ("ability to pay"), algo que afecta especialmente al impuesto sobre la renta.

Como hemos comentado anteriormente, el ejercicio empírico fundamental de la tesis, por falta de información, se basa en la asunción implícita de que el fraude estaba homogéneamente distribuido. Para afrontar esta cuestión, en el cuarto capítulo de la tesis se ha realizado una estimación del fraude en el IRPF, el impuesto principal del nuevo sistema y que debía aportar la mayor progresividad. La hipótesis de partida es que, por resultar más sencilla la ocultación de rentas no salariales, el fraude estaría concentrado en las decilas superiores de contribuyentes y tendría un impacto negativo sobre la progresividad.

Pese a su obvio interés, los estudios sobre la distribución social del fraude son escasos. Esto se debe en buena parte a la falta de datos adecuados: lo preferible, información derivada de inspecciones, sólo ha estado por el momento disponible a gran escala en Estados Unidos (Johns y Slemrod, 2010). Han aparecido, no obstante, aproximaciones alternativas: la explotación de la discrepancia para con la Contabilidad Nacional (Marino y Zizza, 2012; Alm y Borders, 2014), y la estimación econométrica de ratios de cumplimiento utilizando declaraciones fiscales no inspeccionadas (Feldman y Slemrod, 2007). En la tesis, se aplican ambas opciones para el caso de España entre los años 1970 y 2001. La metodología de Feldman 
y Slemrod, además, se modifica ligeramente para introducir una corrección por el sesgo de selección, siguiendo a Heckman (1979). La parte principal de este trabajo se encuentra actualmente en proceso de evaluación editorial y por ello no se pueden compartir los resultados más allá de un breve comentario; los detalles son consultables en Torregrosa Hetland (2015a).

Como se ha comentado en la sección 2, el impuesto sobre la renta moderno (IRPF) se introdujo en España en 1978. Previamente, había existido un abanico de impuestos de producto (dirigidos a cada fuente de renta por separado), a los que se superponía una contribución especial para las rentas más altas (con origen en 1932). Obviamente, este esquema no seguía las ideas de generalidad, tratamiento homogéneo de las rentas, y progresividad, que sí estaban en la base del nuevo IRPF. Las resistencias al cambio fueron agudas: un elevado porcentaje de individuos no presentaba sus declaraciones, y los que lo hicieron declaraban de media importes muy por debajo de su valor real. El nuevo impuesto se vio muy afectado por la falta de cumplimiento y también por las bajas valoraciones legales aplicadas en algunos rendimientos, a pesar del desarrollo administrativo y legal de las décadas siguientes.

Para estimar la magnitud del problema, se ha obtenido en primer lugar una discrepancia agregada como aproximación al fraude en sentido amplio (no exclusivamente legal ${ }^{12}$ ). Utilizamos para ello la ratio entre las magnitudes declaradas en el impuesto (según las estadísticas del IRPF de 1970 y los microdatos fiscales para años posteriores, utilizados ya para la sección 4) y las de la Contabilidad Nacional (siguiendo por tanto el marco general de la tesis, como en la sección 3). Esta discrepancia se muestra en la figura 4.

Las rentas del trabajo eran las más correctamente declaradas, ya en 1971, pero especialmente a partir de los años ochenta ${ }^{13}$. Por otra parte, las rentas del capital tuvieron el peor comportamiento, mientras que las del auto-empleo experimentaron una importante mejora. El total muestra un aumento considerable, pero todavía a finales del periodo el fraude era sin duda un elemento preocupante, pesando cerca de un $20 \%$. 
Figura 4: Cumplimiento por tipo de renta (contribuyentes obligados)

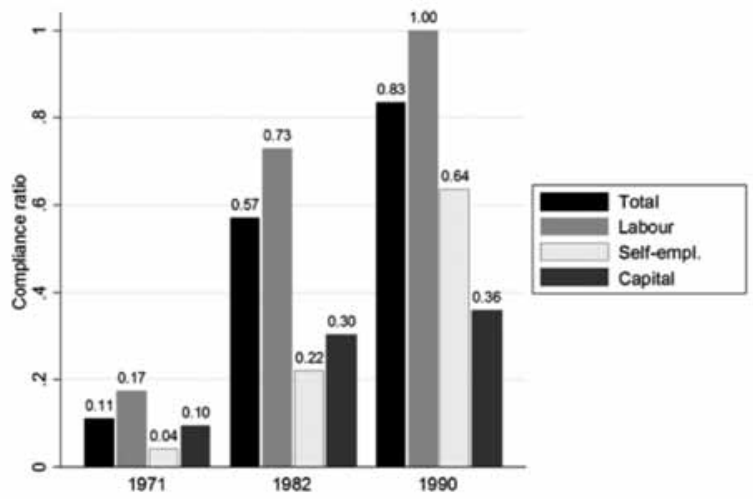

Fuente: Cálculos de la autora (Torregrosa Hetland, 2015a).

Un segundo ejercicio se basó en la re-ponderación de las observaciones de las EPFs para ajustarlas a la población declarante, dejando así el impacto de la no declaración. Con ello se ha realizado un cálculo de cumplimiento por niveles de renta, siguiendo a Matsaganis, Benedek, Flevotomou, Lelkes, Mantovani, y Nienadowska (2010). Se trata de un ejercicio aproximado y con escasa fiabilidad, pero que en cualquier caso muestra, en diversas alternativas, niveles de cumplimiento totales decrecientes con el nivel de renta.

Mi tercera estimación sigue a Feldman y Slemrod (2007) y Domí nguez, López, y Rodrigo (2016), que aplicaron el marco de los primeros al IRPF español en 2008. El método está basado, nuevamente, en la propuesta de Pissarides y Weber (1989): considerando que las rentas del trabajo son las más fiables, se puede estimar un ratio de cumplimiento para los demás tipos de ingresos, en base a su relación con los donativos realizados (que son el equivalente al gasto en alimentación en el modelo inicial). Los resultados indican que las rentas no salariales estarían declaradas aproximadamente en un $40 \%$ de su importe real en 1982. Para 2001, el cumplimiento habría subido ligeramente para las rentas del capital fijo (ahora cerca del 50\%) y del auto-empleo (cerca del 70\%), pero habría descendido en el capital móvil (en torno al 30\%). 
El corolario de estos resultados es que el fraude habría tenido un fuerte impacto negativo sobre la progresividad del impuesto, confirmando nuestra hipótesis de partida. Dada la elevada concentración de la renta en la decila superior de contribuyentes, un cumplimiento del $60-70 \%$ en este nivel suponía un agujero muy preocupante para la capacidad recaudatoria y redistributiva del sistema fiscal. Los indicadores de redistribución y progresividad obtenidos con datos de rentas declaradas estarían sesgados, según unos sencillos cálculos, en un $59-33 \%$ y $44-17 \%$ respectivamente (descendiendo a lo largo de los años considerados). La desigualdad real sería un 10-20\% más alta de lo que aparece, y el tipo medio efectivo un $16-21 \%$ inferior.

Sin fraude, el impuesto sobre la renta se hubiera comportado de manera notablemente distinta, con una presión mucho mayor sobre las rentas altas debido a la aplicación real de la tarifa. Pese a la reducción del problema, todavía en el año 2001 la redistribución derivada del IRPF hubiera sido el doble si la ocultación de rentas se hubiese logrado erradicar.

\section{Comentarios finales}

La aparente contradicción de los resultados previos con lo esperado de una transición a la democracia nos llevó a preguntarnos si la equidad fiscal no era realmente una demanda de la ciudadanía, o hubieron obstáculos insalvables en las instituciones representativas interiores o la economía política internacional.

Una investigación sobre las actitudes sociales en cuanto a la fiscalidad, fundamentalmente en encuestas de opinión y en la prensa, parece apoyar la idea de que la progresividad era un aspecto deseado por la ciudadanía -aunque no una prioridad. Las mayores quejas se dirigen a los aumentos de la presión fiscal sobre los trabajadores, derivados de la inflación, y al fraude. La prensa da voz a algunas opiniones muy favorables al descenso de la desigualdad y a la reforma fiscal progresiva, que no tuvo una alternativa clara en un primer momento; si bien también muestra que dicha alternativa apareció al principio de los ochenta, en el marco de la economía de la oferta. 
¿Hubo un legado institucional de la dictadura que impidió la traslación a la realidad de esta demanda social? Este aspecto ha sido debatido por cientistas políticos, si bien no aplicado directamente a la fiscalidad: Lago y Montero (2005) describen los orígenes del sistema electoral en el marco de la transición, mostrando cómo varios de sus rasgos introdujeron un sesgo hacia la representación de la opinión conservadora, más dominante en los distritos rurales. La importancia de estos mecanismos no debería ser subestimada, puesto que contribuyó a la formación inicial del sistema de partidos, en torno a las primeras elecciones democráticas de 1977: los partidos exitosos lograron acceso al debate constitucional, notoriedad en los medios, y fondos públicos para su actividad.

Otra interpretación posible es la del marco económico. El modelo europeo que se pretendió introducir había sido el fruto de la economía keynesiana de la postguerra, en un marco de crecimiento prolongado y paz social en las democracias occidentales. El nuevo contexto de ralentización del crecimiento está muy vinculado a los cambios en la teoría de las finanzas públicas, y ciertamente dificultó la expansión de los impuestos, especialmente los progresivos. El énfasis en los setenta y ochenta pasó a colocarse en la promoción del ahorro y la inversión privadas.

La apertura internacional reforzó este proceso, al proporcionar a los propietarios del capital una opción "salida" creíble (Bates y Lien, 1985; Freeman y Quinn, 2012). En las últimas décadas del siglo XX, la movilidad del capital y la competencia fiscal son factores fundamentales de la presión contra la progresividad. La apertura económica de Espańa fue en aumento, con el hito de la entrada en la CEE en 1986, y el subsiguiente proceso de construcción del mercado único -que se realizó con una notable armonización de la imposición sobre el consumo, pero sin el mismo esfuerzo en cuanto a la directa.

Podemos terminar buscando algunas respuestas en la comparación internacional. El debate ha tendido a contraponer progresividad y extensión de la redistribución, desde que Steinmo (1989) contrapusiera el sistema sueco, de impuestos relativamente regresivos pero con elevada capacidad recaudatoria para financiar gastos redistributivos, con el 
modelo anglosajón, de impuestos más progresivos pero de menor incidencia. En la misma línea, Lindert (2004) argumentó que históricamente han sido necesarios impuestos regresivos para permitir la financiación de niveles de gasto social elevados. Las bases de esta idea fueron confirmadas empíricamente por Prasad y Deng (2009), así como en la comparación entre los resultados de Piketty y Saez (2007) para EEUU con los de Bengtsson, Holmlund, y Waldenström (2015) para Suecia.

¿Cómo encaja España en este marco? Se podría decir que, al igual que la expansión de los ingresos públicos a finales de los setenta requería incrementar la presión sobre la parte alta de la sociedad, un desarrollo mayor de los niveles de presión fiscal bajo la crisis económica parece haber sido políticamente viable sólo si al mismo tiempo se limitaba la progresividad del sistema. Pero el resultado final, evidenciado en Torregrosa Hetland (2015b), fue que el conjunto de impuestos y transferencias del país no llegó a los niveles de redistribución global que se encuentran en el Reino Unido y Estados Unidos (paradigmas del Estado del Bienestar limitado, liberal). La experiencia de la periferia sur europea puede no encajar completamente en un modelo bipolar. Los rezagados recurrieron también a la imposición regresiva para expandir el gasto social, como los líderes en estas políticas habían hecho. Pero un contexto histórico diferente impidió el establecimiento de sistemas de impuestos y transferencias altamente redistributivos.

\section{BibliografíA}

ACEMOGLU, D., Y J. A. ROBINSON (2001): "A Theory of Political Transitions," American Economic Review, 91(4), 938-963. ACEMOGLU, D., Y J. A. ROBINSON (2008): "Persistence of Power, Elites, and Institutions," American Economic Review, 98(1), 267-93. AIDT, T. S., Y P. S. JENSEN (2009a): "Tax structure, size of government, and the extension of the voting franchise in Western Europe, 18601938," International Tax and Public Finance, 16(3), 362-394.

AIDT, T. S., Y P. S. JENSEN (2009b): “The taxman tools up: An event history study of the introduction of the personal income tax," Journal of Public Economics, 93(1-2), 160-175. 
ALBERTUS, M., Y V. MENALDO (2014): “Gaming Democracy: Elite Dominance during Transition and the Prospects for Redistribution," British Journal of Political Science, 44, 575-603. ALCAIDE, A., Y J. ALCAIDE (1974): "Metodología para la estimación de la distribución personal de la renta en Espańa en 1970," Hacienda Pública Española, (26), 55-63.

ALCAIDE, J. (2000): "La renta nacional de España y su distribución. Serie años 1898 a 1998,” en 1900-2000. Historia de un esfuerzo colectivo: cómo España superó el pesimismo y la pobreza, ed. J. V. Fuertes, pp. 810, 741. Fundación BSCH, Madrid.

ALM, J., Y K. BORDERS (2014): "Estimating the "Tax Gap" at the State Level: The Case of Georgia's Personal Income Tax," Public Budgeting \& Finance, 34(4), 61-79.

ARDANAZ, M., Y C. SCARTASCINI (2013): "Inequality and Personal Income Taxation. The Origins and Effects of Legislative Malapportionment," Comparative Political Studies, 46(12), 1636-1663.

AYALA, L., A. JURADO, Y F. PEDRAJA (2006): “Desigualdad y bienestar en la distribución intraterritorial de la renta, 19732000," Investigaciones Regionales, 8, 5-30.

BANDRÉS, E. (1993): "La eficacia redistributiva de los gastos sociales. Una aplicación al caso español (1980-1990)," en I Simposio sobre Igualdad y Distribución de la Renta y Riqueza, vol. VII, Sector Público y Redistribución, pp. 123-172. Fundación Argentaria, Madrid.

BARNA, T. (1945): Redistribution of income through public finance in 1937. Oxford University Press, Oxford.

BARREIX, A., M. BÈS, Y J. ROCA (eds.) (2009): La equidad fiscal en Centroamérica, Panamá y República Dominicana. BIDEurosocial, Washington.

BARTELS, L. (2005): "Homer gets a tax cut: Inequality and public policy in the American mind," Perspectives on Politics, 3(1), 15-31.

BATES, R. H., Y D.-H. D. LIEN (1985): "A Note on Taxation, Development, and Representative Democracy," Politics and Society, 14, 53-70. 
BENGTSSON, N., B. HOLMLUND, Y D. WALDENSTRÖM (2015): "Lifetime versus Annual Tax Progressivity: Sweden, 1968-2009," Scandinavian Journal of Economics, (forthcoming). BOIX, C. (2003): Democracy and redistribution. Cambridge University Press, Cambridge.

BRECEDA, K., J. RIGOLINI, Y J. SAAVEDRA (2009): “Latin America and the Social Contract: Patterns of Social Spending and Taxation," Population and Development Review, 35(4), 721-748.

CALERO, J. (2001): "La Incidencia distributiva del gasto público social. Análisis general y tratamiento específico de la incidencia distributiva entre grupos sociales y entre grupos de edad," $\mathrm{Pa}-$ peles de Trabajo 20/01, Instituto de Estudios Fiscales, Madrid.

CALVO SOTELO, L. (1990): Memoria viva de la transición. Plaza y Janés, Esplugues de Llobregat (Barcelona).

CEPAL (1991): Magnitud de la pobreza en América Latina en los años ochenta, no. 81 en Estudios e informes de la CEPAL. Santiago de Chile.

COLOMER, J. (1998): La transición a la democracia: el modelo español. Anagrama, Barcelona.

COMÍN, F. (1994): "El fraude fiscal en la Historia: un planteamiento de sus fases," Hacienda Pública Española, I, 31-46.

COMÍN, F. (2007): "Reaching a Political Consensus for Tax Reform in Spain: The Moncloa Pacts, Joining the European Union and the Rest of the Journey," en Fiscal Reform in Spain. Accomplishments and Challenges, ed. J. Martínez Vázquez, y J. F. Sanz Sanz, pp. 8-57. Edward Elgar, Cheltenham, UK.

COMÍN, F. (2010): "Los seguros sociales y el Estado del Bienestar en el siglo XX," en Los origenes del Estado del Bienestar en España, 1900-1945: los seguros de accidentes, vejez, desempleo y enfermedad, ed. J. P. y J. Silvestre, pp. 17-49. Prensas Universitarias de Zaragoza, Zaragoza.

CRUCES, G., R. PEREZ-TRUGLIA, Y M. TETAZ (2013): "Biased perceptions of income distribution and preferences for re- 
distribution: Evidence from a survey experiment," Journal of Public Economics, 98(C), 100-112.

DOMÍNGUEZ, F., J. LÓPEZ, Y F. RODRIGO (2016): “El hueco que deja el diablo: una estimación del fraude en el IRPF con microdatos tributarios," Revista de Economía Aplicada, (forthcoming).

ENGEL, E., A. GALETOVIC, Y C. RADDATZ (1999): “Taxes and income distribution in Chile: some unpleasant redistributive arithmetic," Journal of Development Economics, 59(1), 155-192. ESPING-ANDERSEN, G. (1990): The Three Worlds of Welfare Capitalism. Cambridge University Press, Cambridge.

ESPUELAS, S. (2013): La evolución del gasto social público en España, 1850-2005, vol. 63 of Estudios de Historia Económica. Banco de España, Madrid.

FELDMAN, N. E., Y J. SLEMROD (2007): "Estimating tax noncompliance with evidence from unaudited tax returns," The Economic Journal, 117(518), 327-352.

FIXLER, D., Y D. JOHNSON (2012): "Accounting for the Distribution of Income in the U.S. National Accounts," Paper prepared for the NBER Conference on Research in Income and Wealth "Measuring Economic Stability Progress Conference", September 30.

FREEMAN, J., Y D. QUINN (2012): "The Economic Origins of Democracy Reconsidered," American Political Science Review, 106, 58-80.

FUENTES QUINTANA, E. (1990): Las reformas tributarias en España. Teoría, historia y propuestas. Crítica, Barcelona.

FUENTES QUINTANA, E. (2004): "Los Pactos de la Moncloa y la Constitución de 1978," en Economía y economistas españoles, ed. E. Fuentes Quintana, vol. 8, pp. 163-238. Galaxia Gutenberg: Círculo de Lectores, Barcelona.

GARDE, J., R. MARTÍNEZ, Y J. RUIZ-HUERTA (1996): Los estudios sobre distribución de la renta en España: fuentes, resultados $y$ perspectivas de futuro vol. VI, pp. 257-314. Fundación Argentaria, Madrid. 
GIULIANO, P., Y A. SPILIMBERGO (2009): "Growing Up in a Recession: Beliefs and the Macroeconomy," NBER Working Papers 15321, National Bureau of Economic Research, Inc. GRADSTEIN, M., Y B. MILANOVIC (2004): "Does Libertè = Egalité? A Survey of the Empirical Links between Democracy and Inequality with Some Evidence on the Transition Economies," Journal of Economic Surveys, 18(4), 515-537.

GRÜNER, H. P., Y G. CORNEO (2000): "Social Limits to Redistribution," American Economic Review, 90(5), 1491-1507.

HARMS, P., Y S. ZINK (2003): "Limits to redistribution in a democracy: a survey," European Journal of Political Economy, 19(4), 651-668.

HECKMAN, J. (1979): "Sample selection bias as a specification error," Econometrica, 47(1), 153-61.

INSTITUTO DE ESTUDIOS FISCALES (1973): Informe sobre el sistema tributario español. IEF, Madrid, edition 2002.

INSTITUTO DE ESTUDIOS FISCALES (1976): Sistema tributario español: Criterios para su reforma. IEF, Madrid, edition 2002. JOHNS, A., Y J. SLEMROD (2010): “The Distribution of Income Tax Noncompliance," National Tax Journal, 63, 397-418.

KLEVEN, H. J., C. T. KREINER, Y E. SAEZ (2015): "Why Can Modern Governments Tax So Much? An Agency Model of Firms as Fiscal Intermediaries," .

KORPI, W., Y J. PALME (1998): “The Paradox of Redistribution and Strategies of Equality: Welfare State Institutions, Inequality, and Poverty in the Western Countries," American Sociological Review, 63(5), 661-87.

KUZIEMKO, I., M. I. NORTON, E. SAEZ, Y S. STANTCHEVA (2013): "How Elastic Are Preferences for Redistribution? Evidence from Randomized Survey Experiments," NBER Working Papers 18865, National Bureau of Economic Research, Inc.

LAGO, I., Y J. R. MONTERO (2005): ““Todavía no sé quiénes, pero ganaremos": Manipulación política del sistema electoral español," Zona Abierta, 110/111, 279-348. 
LINDERT, P. (1994): “The Rise of Social Spending, 1880-1930," Explorations in Economic History, 31(1), 1-37.

LINDERT, P. (2004): Growing Public. Cambridge University Press, New York.

MANRESA, A., Y S. CALONGE (2001): "La incidencia impositiva y la redistribución de la renta en Espańa: un análisis empírico," Papeles de Economía Española, 88, 216-229.

MARES, I., Y D. QUERALT (2015): "The Non-Democratic Origins of Income Taxation," Comparative Political Studies, (forthcoming). MARINO, M. R., Y R. ZIZZA (2012): "The personal income tax evasion in Italy : an estimate by taxpayer's type," en Tax Evasion And The Shadow Economy, ed. M. Pickhardt, y A. Prinz, pp. 33-60. Edward Elgar, Cheltenham, UK.

MARTIN, I. W., A. K. MEHROTRA, Y M. PRASAD (2009): The new fiscal sociology: Taxation in comparative and historical perspective. Cambridge University Press.

MARTÍNEZ-LÓPEZ, D. (2013): "The underreporting of income by self-employed workers in Spain," SERIEs, 4(4), 353-371.

MATSAGANIS, M., D. BENEDEK, M. FLEVOTOMOU, O. LELKES, D. MANTOVANI, Y S. NIENADOWSKA (2010): "Distributional implications of income tax evasion in Greece, Hungary and Italy," MPRA Working Paper 21465.

MELTZER, A., Y S. RICHARD (1981): "A Rational Theory of the Size of Government," Journal of Political Economy, 89, 914-927. MILANOVIC, B., P. H. LINDERT, Y J. G. WILLIAMSON (2011): "Pre-Industrial Inequality," Economic Journal, 121(551), 255-272. MOLINERO, C., Y P. YSÀS (2008): La anatomía del franquismo: de la supervivencia a la agonía: 1945-1977. Crítica, Barcelona. MUSGRAVE, R. A. (1951): "Distribution of tax payments by income groups: a case study for 1948," National Tax Journal, 4, 1-53.

NERI, A., Y R. ZIZZA (2010): "Income reporting behaviour in the SHIW," Temi di Discussione 777, Bank of Italy.

OLIVER, J. (1997): "Ingreso, consumo y ahorro de las familias: propuesta de una metodología para la explotación de la Encuesta de Presupuestos Familiares," Documentos de trabajo, FIES. 
ONRUBIA, J., M. C. RODADO, S. DÍAZ, Y C. PÉREZ (2007): "Progresividad y redistribución a través del IRPF español: Un análisis de bienestar social para el periodo 1982-1998," Hacienda Pública Española, 183, 81-124.

PAN-MONTOJO, J. L. (1996): "Una larga e inconclusa transición: la reforma tributaria, 1977-1986," en Historia de la transición. 19751986, ed. J. Tusell, y A. Soto, pp. 264-304. Alianza, Madrid.

PEACOCK, A. T., Y J. WISEMAN (1961): The Growth of Public Expenditure in the United Kingdom. Princeton University Press, Cambridge, MA. PENA, J. B., Y F. J. CALLEALTA (1996): Distribución personal de la renta en España: corrección y modelización de la información básica: desigualdad y análisis. Pirámide, Madrid.

PERONA, D. (1972): La distribución de la carga tributaria en Espana. Instituto de Estudios Fiscales, Madrid.

PIKETTY, T., Y E. SAEZ (2007): "How Progressive Is the U.S. Federal Tax System? A Historical and International Perspective," Journal of Economic Perspectives, 21(1), 3-24.

PISSARIDES, C. A., Y G. WEBER (1989): "An Expenditure-Based Estimate of Britain's Black Economy," Journal of Public Economics, 39(1), 17-32.

PRASAD, M., Y Y. DENG (2009): "Taxation and the worlds of welfare," Socio-Economic Review, 7, 431-457.

SAZ, I. (2010): "La lucha por la libertad en España desde una perspectiva comparada (1962-1977)," en Novísima: II Congreso Internacional de Historia de Nuestro Tiempo, ed. C. Navajas, y D. Iturriaga, pp. 71-80.

SCHEVE, K., Y D. STASAVAGE (2012): "Democracy, War, and Wealth: Lessons from Two Centuries of Inheritance Taxation," American Political Science Review, 106(1), 81-102.

STEINMO, S. (1989): "Political Institutions and Tax Policy in the United States, Sweden, and Britain," World Politics, 41(4), 500-535.

SVALLFORS, S. (2013): “Government quality, egalitarianism, and attitudes to taxes and social spending: a European comparison," European Political Science Review, 5, 363-380. 
TIMMONS, J. (2005): “The Fiscal Contract: States, Taxes, and Public Services," World Politics, 57, 530-576.

TORREGROSA HETLAND, S. (2015a): "Bypassing progressive taxation: fraud and base erosion in the Spanish income tax, 1970-2001," IEB Working Papers, 2015/31.

TORREGROSA HETLAND, S. (2015b): "Did Democracy bring Redistribution? Insights from the Spanish tax system (19601990)," European Review of Economic History, 3(19), 294-315. TORREGROSA HETLAND, S. (2016): "Sticky income inequality in the Spanish transition (1973-1990)," Revista de Historia Económica / Journal of Iberian and Latin American Economic History, 34(1), 39-80.

WILENSKY, H. L. (2002): Rich Democracies: Political Economy, Public Policy, and Performance. University of California Press, Berkeley.

\section{Notas de Página}

${ }^{1}$ Esta nota de investigación es un resumen de la tesis de Doctorado en Historia Económica "Tax system and redistribution: the Spanish fiscal transition (1960-1990)", realizada en la Universidad de Barcelona bajo supervisión de Alfonso Herranz Loncán y Alejandro Esteller Moré, a quienes agradezco la ayuda recibida. Mi trabajo contó con apoyo financiero del programa FPU del Ministerio de Educación de Espańa, así como del Proyecto de Investigación ECO201239169-C03-03.

${ }^{2}$ En palabras de L. Calvo Sotelo, primer ministro en 198182: "Fernández Ordóńez y su reforma fiscal atrajeron muy pronto sobre UCD las iras de la derecha económica: 'Estáis haciendo política de izquierda con los votos de la derecha'" (Calvo Sotelo, 1990, p. 163).

${ }^{3}$ En este apartado se trata siempre de la desigualdad en la renta disponible, la definición más usual en los estudios comparables (aunque se podría argumentar la conveniencia de utilizar un concepto de renta neta de todos los impuestos, incluidos los indirectos). 
${ }^{4} \mathrm{El}$ factor para el auto-empleo resulta de multiplicar el del trabajo (obtenido por la ratio de discrepancia con CNE) por el resultado relativo de la regresión à la Pissarides y Weber (1989).

${ }^{5}$ Las rentas equivalentes se obtienen utilizando la escala OCDE, que da ponderación $1 \mathrm{al}$ primer adulto del hogar, 0,7 a los siguientes, y 0,5 a los demás miembros por debajo de 14 ańos. El uso de este indicador implica asumir, además, una distribución equitativa de los recursos en el hogar. La obtención de niveles de desigualdad ligeramente inferiores con este cálculo es de esperar, pues los hogares de mayor tamaño tienden a tener rentas más elevadas, siendo una parte de la explicación el número de sus miembros.

${ }^{6}$ Las transferencias totales pasaron del 14 al $25 \%$ de la renta disponible de los hogares, mientras que las rentas del capital lo hicieron del 4 al 7\%. Este último tipo de ingreso está muy concentrado: ausente en las clases inferiores, supone más del $10 \%$ de la renta para la decila superior y alrededor del $30 \%$ en el percentil máximo (de manera similar a otros países).

7"Voting for Welfare? Comparative performance in Ibero-American Democratizations", junto con Cristián Ducoing (Universidad de Umeå).

${ }^{8}$ En el caso de 1982, debido a los microdatos fiscales de IRPF: "PANEL PURO Y EXTENDIDO IRPF 1982/1998 IEF-AEAT (Declarantes)", que se han utilizado también para 1990.

${ }^{9}$ Aunque este tema es discutible, se ha considerado que por las características del sistema de la Seguridad Social en España está justificado tratar las cotizaciones como impuestos y las pensiones como transferencias.

${ }^{10}$ Una estimación alternativa ordena a los hogares según sus rentas brutas más transferencias, para atender al posible carácter mixto de las pensiones.

${ }^{11}$ Los tipos medios efectivos se calculan como la ratio entre el impuesto pagado y la renta utilizada como base (bruta); se diferencian de los legales por la existencia de deducciones, distintas definiciones de la base imponible, etc. La progresividad es el incremento del tipo impositivo (efectivo) cuando aumenta la renta, 
mientras que redistribución se refiere a la diferencia en la desigualdad causada por los impuestos.

${ }^{12}$ Nos referimos a tres fenómenos distintos: la no declaración por parte de individuos obligados a hacerla, las valoraciones legales por debajo del valor económico real, y la ocultación total o parcial de rendimientos por parte de los contribuyentes.

${ }^{13}$ Los índices obtenidos para 1971 se refieren exclusivamente a la declaración de rendimientos en el impuesto antecedente al IRPF, y no a los impuestos de producto.

Fecha de Recepción del Artículo: 11 de abril de 2016 Fecha de Aprobación: 23 de mayo de 2016 